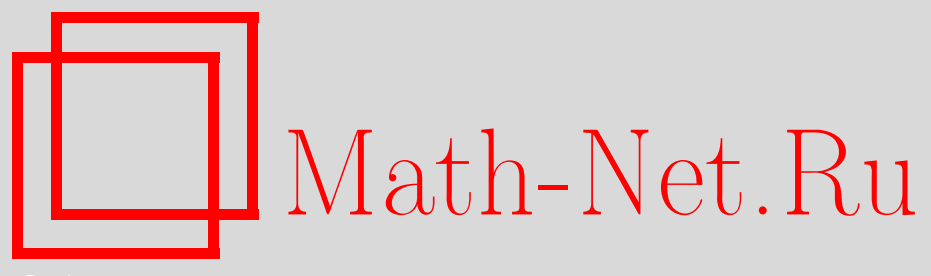

Д. Б. Баралич, В. Н. Груйич, Квазиторические многообразия и малые накрытия над правильно окрашенными многогранниками: вложения и погружения, Матем. сб., 2016, том 207, номер 4, 3-14

DOI: https://doi.org/10.4213/sm8401

Использование Общероссийского математического портала Math-Net.Ru подразумевает, что вы прочитали и согласны с пользовательским соглашением http://www . mathnet.ru/rus/agreement

Параметры загрузки:

IP: 18.234 .156 .22

26 апреля 2023 г., 16:17:15

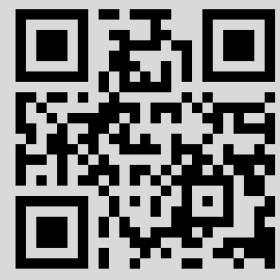




\author{
Д. Б. Баралич, В. Н. Груйич
}

\title{
Квазиторические многообразия и малые накрытия над правильно окрашенными многогранниками: вложения и погружения
}

\begin{abstract}
Строятся малые накрытия и квазиторические многообразия над простыми $n$-мерными многогранниками, допускающими правильную раскраску гиперграней в $n$ цветов. Вычисляются классы Штифеля-Уитни этих многообразий как препятствия к их вложению в евклидовы пространства. Самая большая размерность, необходимая для вложения, достигается в случае, если $n$ - степень двойки.
\end{abstract}

Библиография: 11 названий.

Ключевые слова: вложения, квазиторические многообразия, простые многогранники, раскраски, классы Штифеля-Уитни.

DOI: $10.4213 / \mathrm{sm} 8401$

\section{§ 1. Введение}

1.1. Раскраски простых многогранников. Выпуклый $n$-мерный многогранник простой, если в каждой его вершине сходятся в точности $n$ гиперграней. Правильной раскраской простого $n$-мерного многогранника $P^{n}$ в $k$ цветов называется отображение

$$
h:\left\{F_{1}, \ldots, F_{m}\right\} \rightarrow\{1, \ldots, k\}
$$

его множества гиперграней такое, что $h\left(F_{i}\right) \neq h\left(F_{j}\right)$ для любых двух пересекающихся гиперграней. Хроматическое число $\chi\left(P^{n}\right)$ простого многогранника $P^{n}$ - это наименьшее число $k$ такое, что существует правильная раскраска многогранника $P^{n}$ в $k$ цветов. Хроматические числа симплекса $\Delta^{n}$, куба $I^{n}$ и пермутоэдра $\Pi^{n}$ равны

$$
\chi\left(\Delta^{n}\right)=n+1, \quad \chi\left(I^{n}\right)=n, \quad \chi\left(\Pi^{n}\right)=n .
$$

Очевидно, что для каждого простого многогранника $\chi\left(P^{n}\right) \geqslant n$. Хроматическое число многоугольника равно 2 или 3 в зависимости от четности числа его граней. По знаменитой теореме четырех красок хроматические числа 3 -мерных простых многогранников равны 3 или 4 . В общем случае, если $n \geqslant 4$, не всегда верно $\chi\left(P^{n}\right) \leqslant n+1$. Более того, существуют простые многогранники такие, что их хроматические числа в точности равны числам их гиперграней. Циклические многогранники с $m$ вершинами $C^{n}(m)$ являются примерами таких многогранников (см. [1; пример 0.6]).

Работа выполнена при поддержке Министерства науки Республики Сербии (грант № 174020 и грант № 174034) и Министерства науки Республики Сербской, Боснии и Герцеговини (грант № 19/6-020/961-120/14). 

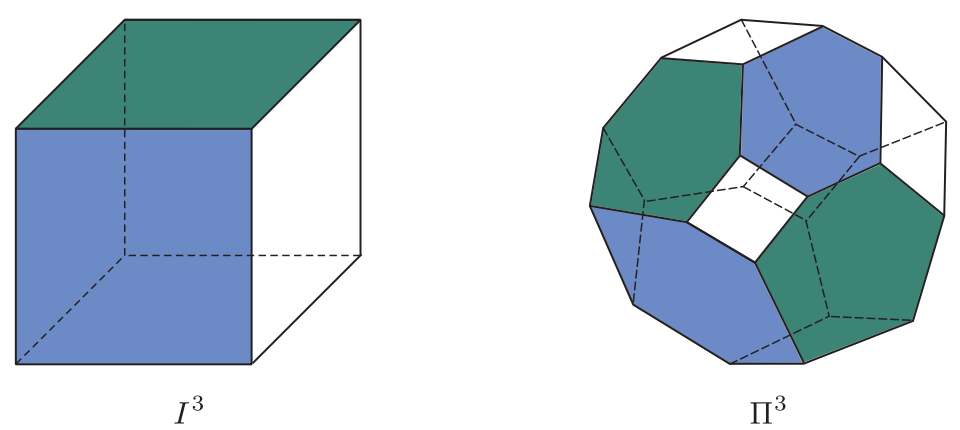

Рис. 1. Раскраски куба и пермутоэдра

Мы рассматриваем класс $n$-мерных простых многогранников, допускающих правильную раскраску в $n$ цветов. Обозначим этот класс через $\mathscr{C}$. Класс $\mathscr{C}$ замкнут по отношению к произведениям (см. [2; конструкция 1.9]) и связным суммам (см. [2; конструкция 1.10]). Также из каждого простого многогранника $P^{n}$ срезанием по всем его граням получается простой многогранник $Q^{n}$, принадлежающий классу $\mathscr{C}$. Полное описание класса $\mathscr{C}$ получено в [3]. Простой $n$-многогранник $P^{n}$ допускает правильную раскраску в $n$ цветов тогда и только тогда, когда любая его двумерная грань имеет четное число ребер (см. [3; теорема 16]).

1.2. Малые накрытия и квазиторические многообразия. Квазиторические многообразия и их вещественные аналоги - малые накрытия введены М. Дэвисом и Т. Янушкиевичем в [4]. Их геометрические и алгебраические топологические свойства близко связаны с комбинаторикой простых многогранников. Следующие определения и понятия подробно изложены в книге [2]. Обозначим

$$
G_{d}=\left\{\begin{array}{ll}
S^{0}, & d=1, \\
S^{1}, & d=2,
\end{array} \quad R_{d}=\left\{\begin{array}{ll}
\mathbb{Z}_{2}, & d=1, \\
\mathbb{Z}, & d=2,
\end{array} \quad \mathbb{K}_{d}= \begin{cases}\mathbb{R}, & d=1 \\
\mathbb{C}, & d=2\end{cases}\right.\right.
$$

где $S^{0}=\{-1,+1\}$ и $S^{1}=\{z|| z \mid=1\}$ есть мультипликативные подгруппы вещественных, соответственно комплексных чисел, $\mathbb{Z}$ - кольцо целых чисел и $\mathbb{Z}_{2}=\{0,1\}$ есть кольцо вычетов по модулю два. Действие группы $G_{d}^{n}$ на пространстве $\mathbb{K}_{d}^{n}$, определяемое как $\left(t_{1}, \ldots, t_{n}\right) \cdot\left(x_{1}, \ldots, x_{n}\right)=\left(t_{1} x_{1}, \ldots, t_{n} x_{n}\right)$, называется стандартным. Пусть $M^{d n}$ - гладкое $d n$-мерное $G_{d}^{n}$-многообразие. Действие группы $G_{d}^{n}$ на многообразие $M^{d n}$ является локально стандартным, если каждая точка из $M^{d n}$ обладает $G_{d}^{n}$-инвариантной окрестностью слабо эквивариантно диффеоморфной некоторому открытому $G_{d}^{n}$-инвариантному подмножеству в $\mathbb{K}_{d}^{n}$ со стандартным действием группы $G_{d}^{n}$. Напомним, что два $G_{d}^{n}$ многообразия $M_{1}^{d n}$ и $M_{2}^{d n}$ называются слабо эквивариантно диффеоморфными, если существуют автоморфизм $\omega: G_{d}^{n} \rightarrow G_{d}^{n}$ и диффеоморфизм $f: M_{1}^{d n} \rightarrow M_{2}^{d n}$ такие, что

$$
f(g \cdot x)=\omega(g) \cdot f(x)
$$


для любых $g \in G_{d}^{n}$ и $x \in M_{1}^{d n}$. Гладкое $d n$-мерное $G_{d}^{n}$-многообразие $M^{d n}$ с локально стандартным действием группы $G_{d}^{n}$, таковым что пространство орбит $M^{d n} / G_{d}^{n}$ диффеоморфно как многообразие с углами простому $n$-многограннику $P^{n}$, называется малым накрытием над $P^{n}$, если $d=1$, соответственно квазиторическим многообразием над $P^{n}$, если $d=2$. Пусть $\pi:{ }^{d n} \rightarrow P^{n}$ есть проекция и $\left\{F_{1}, \ldots, F_{m}\right\}$ - множество гиперграней многогранника $P^{n}$. Прообразы $M_{j}^{d(n-1)}=\pi^{-1}\left(F_{j}\right)$ являются $G_{d}^{n}$-инвариантными подмногообразиями коразмерности $d$, называемыми характеристическими подмногообразиями. Каждому характеристическому подмногообразию $M_{j}^{d(n-1)}$ соответствует стационарная подгруппа $G\left(F_{j}\right)=G_{\lambda_{j}}$ ранга один, где $G_{\lambda_{j}}=\left\{(1, \ldots, 1),\left((-1)^{\lambda_{1 j}}, \ldots\right.\right.$, $\left.\left.(-1)^{\lambda_{n j}}\right)\right\}$ для $\lambda_{j}=\left(\lambda_{1 j}, \ldots, \lambda_{n j}\right) \in \mathbb{Z}_{2}^{n} \backslash\{0\}$, если $d=1$ и $G_{\lambda_{j}}=\left\{\left(e^{2 \pi i \lambda_{1 j} t}, \ldots\right.\right.$, $\left.\left.e^{2 \pi i \lambda_{n j} t}\right) \mid t \in \mathbb{R}\right\}$ для некоторого примитивного вектора $\lambda_{j}=\left(\lambda_{1 j}, \ldots, \lambda_{n j}\right) \in \mathbb{Z}^{n}$, определенного с точностью до знака, если $d=2$. Таким образом, действие группы $G_{d}^{n}$ на многообразие $M^{d n}$ определяет характеристическое отображение

$$
l_{d}:\left\{F_{1}, \ldots, F_{m}\right\} \rightarrow R_{d}^{n}
$$

сопоставляющее гиперграни $F_{j}$ многогранника $P^{n}$ некоторый примитивный вектор $\lambda_{j}=\left(\lambda_{1 j}, \ldots, \lambda_{n j}\right) \in R_{d}^{n}$. Обозначим через $\Lambda=\left(\lambda_{1}, \ldots, \lambda_{m}\right)$ матрицу этих векторов. Тогда $\operatorname{det} \Lambda_{(V)}= \pm 1$ для каждой вершины $V \in P^{n}$, где $\Lambda_{(V)}$ - подматрица матрицы $\Lambda$, образованная векторами, соответствующими гиперграням, пересекающимся в этой вершине. Назавем пару $\left(P^{n}, \Lambda\right)$, удовлетворяющую указанному условию на подматрицы, характеристической naрой. Из характеристической пары $\left(P^{n}, \Lambda\right)$ многообразие $M^{d n}$ восстанавливается с точностью до слабого эквивариантного диффеоморфизма следующим образом (см. [4] и [2; конструкция 6.18]). Пусть $F_{i_{1}} \cap \cdots \cap F_{i_{k}}$ - наименьшая грань, содержащая $q \in P^{n}$. Сопоставим точке $q$ подгруппу $G(q)=G_{\lambda_{i_{1}}} \times \cdots \times G_{\lambda_{i_{k}}}$. Положим $M\left(P^{n}, \Lambda\right)=G_{d}^{n} \times P^{n} / \sim$, где $\left(t_{1}, p\right) \sim\left(t_{2}, q\right)$, тогда и только тогда, когда $p=q$ и $t_{1} t_{2}^{-1} \in G(q)$.

Хотя не каждый простой многогранник допускает характеристическое отображение (см. [4; пример 1.15]), рассматриваемым многогранникам $P^{n} \in \mathscr{C}$ можно простым способом сопоставить характеристическую матрицу $\Lambda$, если каждый цвет $i \in\{1, \ldots, n\}$ отождествим с вектором $e_{i}$ стандартного базиса в $R_{d}^{n}$.

Кольцо когомологий малого накрытия и квазиторического многообразия $M^{d n}, d=1,2$, описывается следующим образом. Пусть $v_{j}=D\left[M_{j}^{d(n-1)}\right]$, $j=1, \ldots, m,-$ когомологические классы, двойственные по Пуанкаре к фундаментальным классам характеристических подмногообразий. Характеристическая матрица $\Lambda=\left(\lambda_{1}, \ldots, \lambda_{m}\right)$ определяет следующие линейные формы

$$
\theta_{i}:=\sum_{j=1}^{m} \lambda_{i j} v_{j}, \quad i=1, \ldots, n,
$$

где $\lambda_{j}=\left(\lambda_{1 j}, \ldots, \lambda_{n j}\right)^{t} \in R_{d}^{n}, j=1, \ldots, m$. Пусть $\mathscr{J}-$ идеал в $R_{d}\left[v_{1}, \ldots, v_{m}\right]$, порожденный элементами $\theta_{1}, \ldots, \theta_{n}$, и $\mathscr{I}$ - идеал Стенли-Райснера многогранника $P^{n}$, порожденный такими мономами $v_{i_{1}} \cdots v_{i_{k}}$, что $F_{i_{1}} \cap \cdots \cap F_{i_{k}}=\varnothing$ в $P^{n}$, 
$i_{1}<\cdots<i_{k}$. Тогда для кольца когомологий имеет место изоморфизм (см. [4])

$$
H^{*}\left(M^{d n}, R_{d}\right) \simeq R_{d}\left[v_{1}, \ldots, v_{m}\right] /(\mathscr{I}+\mathscr{J}) .
$$

Формула (1.1) верна при $d=2$ и с коэффициентами в $\mathbb{Z}_{2}$. Полный класс Штифеля-Уитни задается следующей формулой Дэвиса-Янушкиевича:

$$
w\left(M^{d n}\right)=\prod_{i=1}^{m}\left(1+v_{i}\right) \in H^{*}\left(M^{d n} ; \mathbb{Z}_{2}\right),
$$

где в случае $d=2$ классы $v_{i}$ представляют $\mathbb{Z}_{2}$-ограничения соответствующих целочисленных классов.

1.3. Вложения и погружения. Вложения и погружения многообразий есть классический вопрос алгебраической и дифференциальной топологии. Мы рассматриваем вложения и погружения в гладкой категории. Пусть для многообразия $M^{n}$ числа $\operatorname{imm}\left(M^{n}\right)$ и $\operatorname{em}\left(M^{n}\right)$ определены как наименьшие размерности евклидовых пространств, в которые это многообразие погружается, соответствено вкладывается. Согласно теореме Уитни для каждого гладкого многообразия $M^{n}$ выполнено $\operatorname{imm}\left(M^{n}\right) \leqslant 2 n-1$ и $\mathrm{em}\left(M^{n}\right) \leqslant 2 n$. С другой стороны, нормальные классы Штифеля-У итни $\bar{w}_{i}$ являются препятствиями к вложениям и погружениям многообразий в евклидовы пространства. Напомним, что нормальные классы Штифеля-Уитни $\bar{w}_{i}\left(M^{n}\right)$ являются характеристическими классами стабильного нормального расслоения многообразия $M^{n}$.

ТеОрема 1 (см. [5]). Пусть для гладкого многообразия $M^{n}$ определено число $k=\max \left\{i \mid \bar{w}_{i}\left(M^{n}\right) \neq 0\right\}$. Тогда $\operatorname{imm}\left(M^{n}\right) \geqslant n+k u \operatorname{em}\left(M^{n}\right) \geqslant n+k+1$.

Классы Штифеля-Уитни являются также препятствиями к так называемым вполне косым вложениям, введенным М. Гоми и С. Табачниковым в [6]. Многообразие $M^{n}$ вполне косо вкладывается в евклидово пространство $\mathbb{R}^{N}$, если любые две касательные прямые в различных точках многообразия $M^{n}$ являются скрещивающимися прямыми в $\mathbb{R}^{N}$. В работе [6] доказано, что для числа $N\left(M^{n}\right)$, определенного как наименьшая размерность евклидовых пространств, в которые многообразие $M^{n}$ вполне косо вкладывается, выполнено

$$
2 n+2 \leqslant N\left(M^{n}\right) \leqslant 4 n+1 .
$$

Более точное ограничение снизу получено в [7].

ТЕОРема 2 (см. [7; предложение 1, следствие 4]). Если

$$
k=\max \left\{i \mid \bar{w}_{i}\left(M^{n}\right) \neq 0\right\},
$$

$\operatorname{mo} N\left(M^{n}\right) \geqslant 2 n+2 k+1$.

Вложения и погружения квазиторических многообразий над кубами изучались в [8].

В статье доказываются следующие результаты. 
Теорема 3. Пусть $n$ - степенъ двойки и $P^{n}$ - простой выпуклый $n$-мерный многогранник, допускающий правильную раскраску в $n$ иветов.

(1) Существует малое накрытие $M^{n}$ над многогранником $P^{n}$, удовлетворяющее равенствам $\operatorname{imm}\left(M^{n}\right)=2 n-1 u \operatorname{em}\left(M^{n}\right)=2 n$.

(2) Существует квазиторическое многообразие $M^{2 n}$ над многогранником $P^{n}$, удовлетворяющее неравенствам $\operatorname{imm}\left(M^{2 n}\right) \geqslant 4 n-2 u \operatorname{em}\left(M^{2 n}\right) \geqslant 4 n-1$, причем при $n \geqslant 3$ оба неравества обращаются в равенства.

Мы построим многообразия $M^{n}$ и $M^{2 n}$ из теоремы 3 явным образом. Используя комбинаторные свойства простого многогранника $P^{n}$ и формулы ДэвисаЯнушкиевича (1.1) и (1.2), вычисляем кольца когомологий $H^{*}\left(M^{d n}, \mathbb{Z}_{2}\right), d=$ 1,2, и классы Штифеля-Уитни $w_{k}\left(M^{d n}\right), d=1,2$. В случае когда $n$ является степенью двойки, мы покажем, что классы $\bar{w}_{d(n-1)}\left(M^{d n}\right), d=1,2$, ненулевые. Это утверждение доказывает теорему 3. Также из этого утверждения и теоремы 2 для вполне косых вложений построенных многообразий немедленно вытекает

СлЕДСтвиЕ 1. Если $n$ - степенъ двойки, то

$$
N\left(M^{n}\right) \geqslant 4 n-1, \quad N\left(M^{2 n}\right) \geqslant 8 n-3 .
$$

Получаемые малые накрытия $M^{n}$ являются новым классом многообразий, для которых число $N\left(M^{n}\right)$ равно $4 n-1,4 n$ или $4 n+1$. До сих пор только вещественные проективные пространства являлись примерами многообразий с этим свойством (см. [7]).

В 1985 г. в работе [9] Р.Л. Коэн положительно разрешил знаменитую гипотезу о вложения , доказывая, что каждое замкнутое $n$-многообразие для $n>1$ вкладывается в $\mathbb{R}^{2 n-\alpha(n)}$, где $\alpha(n)$ - количество единиц в двоичной записи числа $n$. Произведения вещественных проективных пространств являются примерами многообразий, размерности которых достигают ограничения сверху. Мы построим новые примеры такого вида в классе малых накрытий.

Теорема 4. Для каждого $n$ существуют малое накрытие $M^{n}$ и квазиторическое многообразие $M^{2 n}$ над некоторым простым $n$-мерным многогранником, допускающим правильную раскраску в п иветов, для которых выполнено

$$
\begin{array}{cl}
\operatorname{imm}\left(M^{n}\right)=2 n-\alpha(n), & \operatorname{imm}\left(M^{2 n}\right) \geqslant 4 n-2 \alpha(n), \\
\mathrm{em}\left(M^{n}\right)=2 n-\alpha(n)+1, & \operatorname{em}\left(M^{2 n}\right) \geqslant 4 n-2 \alpha(n)+1, \\
N\left(M^{n}\right) \geqslant 4 n-2 \alpha(n)+1, & N\left(M^{n}\right) \geqslant 8 n-4 \alpha(n)+1 .
\end{array}
$$

Мы предполагаем, что утверждение теоремы 4 выполнено для каждого простого $n$-мерного многогранника, правильно окрашенного в $n$ цветов.

ГиПотеЗА 1. Пусть $P^{n}$ - простой выпуклый $n$-мерный многогранник, гиперграни которого допускают правильную раскраску в $n$ цветов. Тогда существуют малое накрытие $M^{n}$ и квазиторическое многообразие $M^{2 n}$ над многогранником $P^{n}$, удовлетворяющие неравенствам

$$
\begin{array}{cl}
\operatorname{imm}\left(M^{n}\right)=2 n-\alpha(n), & \operatorname{em}\left(M^{n}\right)=2 n-\alpha(n)+1, \\
\operatorname{imm}\left(M^{2 n}\right) \geqslant 4 n-2 \alpha(n), & \operatorname{em}\left(M^{2 n}\right) \geqslant 4 n-2 \alpha(n)+1 .
\end{array}
$$




\section{§ 2. Многообразия $M^{d n}$}

2.1. Конструкция. Пусть $P^{n}$ - простой многогранник такой, что хроматическое число $\chi\left(P^{n}\right)=n$ и $h:\left\{F_{1}, \ldots, F_{m}\right\} \rightarrow\{1, \ldots, n\}-$ его правильная раскраска. Обозначим через $\mathscr{F}_{j}$ множество $h^{-1}(j)$. Каждая вершина $V \in P^{n}$ является пересечением $n$ различно окрашенных гиперграней. Выберем произвольную вершину $V=H_{1} \cap \cdots \cap H_{n}$, где гипергрань $H_{i}$ окрашена цветом $i$. Назначим каждой гиперграни $H_{i}$ вектор $\lambda_{i}=(\underbrace{0, \ldots, 0}_{i-1}, \underbrace{1, \ldots, 1}_{n-i+1})^{t}$ и векторы $\lambda_{F}=(\underbrace{0, \ldots, 0}_{i-1}, 1, \underbrace{0, \ldots, 0}_{n-i})^{t}$ остальным гиперграням $F \in \mathscr{F}_{i} \backslash\left\{H_{i}\right\}$. Соответствующая матрица $\Lambda$ очевидно производит характеристическое отображение, потому что $\operatorname{det} \Lambda_{(V)}=1$ для каждой вершины $V \in P^{n}$. Определим $M^{d n}=M\left(P^{n}, \Lambda\right)$ как многообразие, восстанавливающееся по характеристической паре $\left(P^{n}, \Lambda\right)$.

2.2. Кольцо когомологий. Пусть $u_{1}, \ldots, u_{n}$ - классы, двойственные по Пуанкаре характеристическим подмногообразиям, соответствующим гиперграням $H_{1}, \ldots, H_{n}$. Для каждой гиперграни $F$ многогранника $P^{n}$, кроме $H_{1}$, $\ldots, H_{n}$, пусть $v_{F}$ обозначает класс, двойственный по Пуанкаре характеристическому подмногообразию над $F$.

Кольцо когомологий многообразия $M^{d n}$ определяется идеалом Стенли-Райснера $\mathscr{I}$ многогранника $P^{n}$ и идеалом $\mathscr{J}$, порожденным линейными формами

$$
\theta_{i}:=\sum_{j=1}^{i} u_{j}+\sum_{F \in \mathscr{F}_{i} \backslash\left\{H_{i}\right\}} v_{F}, \quad i=1, \ldots, n .
$$

Напомним, что

$$
H^{*}\left(M^{d n} ; \mathbb{Z}_{2}\right)=\mathbb{Z}_{2}\left[u_{1}, \ldots, u_{n}, v_{F} \mid F \in \mathscr{F} \backslash\left\{H_{1}, \ldots, H_{n}\right\}\right] /(\mathscr{I}+\mathscr{J}) .
$$

Из раскраски многогранника $P^{n}$ легко следует

ПреДЛОЖениЕ 1. Для гиперграней $F, G \in \mathscr{F}_{i} \backslash\left\{H_{i}\right\}, F \neq G$ соответствующие классы удовлетворяют следующему соотношению в кольще когомологии $H^{*}\left(M^{d n} ; \mathbb{Z}_{2}\right)$ :

$$
v_{F} v_{G}=u_{i} v_{F}=u_{i} v_{G}=0 .
$$

Из предложения 1 и (2.1) выводим

ПреДЛОЖеНИЕ 2. Следующие равенства выполнены в $H^{*}\left(M^{d n} ; \mathbb{Z}_{2}\right)$ :

$$
u_{1}^{2}=0, \quad u_{2}^{2}=u_{1} u_{2}, \quad \ldots, \quad u_{n}^{2}=u_{1} u_{n}+\cdots+u_{n-1} u_{n} .
$$

Пусть $F$ - одна из гиперграней многограника $P^{n}$ и $v_{F}-$ соотвествующий класс над $F$, даже если $F$ является одной из $H_{1}, \ldots, H_{n}$.

Лемма 1. Пусть $k$ - положительное целое число $u h$ - правильная раскраска многогранника $P^{n}$. Тогда класс $v_{F}^{k}$ нулевой или равен однородному многочлену $Q_{k}^{F}\left(u_{1}, \ldots, u_{h(F)-1}, v_{F}\right)$ степени $k$, одночлены которого не содержат квадратов. 
ДокАЗАтЕльство. Пусть $h(F)=i$. Утверждение доказываем индукцией по $k$. Для $k=1$ утверждение очевидно. Предположим, что $v_{F}^{k}=Q_{k}^{F}\left(u_{1}, \ldots\right.$, $\left.u_{i-1}, v_{F}\right)$. Умножая на $v_{F}^{k}$ соотношение

$$
\sum_{j=1}^{i} u_{j}+\sum_{G \in \mathscr{F}_{i} \backslash\left\{H_{i}\right\}} v_{G}=0
$$

и применяя предложение 1 , получаем

$$
v_{F}^{k+1}=\left(u_{1}+\cdots+u_{i-1}\right) Q_{k}^{F}\left(u_{1}, \ldots, u_{i-1}, v_{F}\right) .
$$

Если $v_{F}^{k+1}=0$, утверждение следует непосредственно. В противном случае очевидно, что $\left(u_{1}+\cdots+u_{i-1}\right) Q_{k}^{F}\left(u_{1}, \ldots, u_{i-1}, v_{F}\right)$ есть однородный многочлен степени $k+1$ от переменных $u_{1}, \ldots, u_{i-1}, v_{F}$. Но из соотношений $(2.2)$ следует, что

$$
\left(u_{1}+\cdots+u_{i-1}\right) Q_{k}^{F}\left(u_{1}, \ldots, u_{i-1}, v_{F}\right)=Q_{k+1}^{F}\left(u_{1}, \ldots, u_{i-1}, v_{F}\right),
$$

где все одночлены многочлена $Q_{k+1}^{F}$ свободны от квадратов.

Из леммы 1 немедленно вытекает

СлеДСТвИЕ 2. Каждый класс $v_{F_{i_{1}}}^{r_{1}} \cdots v_{F_{i_{k}}}^{r_{k}}$, не являющийся нулевым, равен однородному многочлену степени $r_{1}+\cdots+r_{k}$ от переменных $u_{1}, \ldots, u_{n}, v_{F_{i_{1}}}$, $\ldots, v_{F_{i_{k}}}$, все одночлены которого свободны от квадратов.

ПРеДЛОЖеНИЕ 3. Класс $u_{1} \cdots u_{n}$ является фундаменталъным когомологическим классом в кольще $H^{d n}\left(M^{d n} ; \mathbb{Z}_{2}\right)$.

ДокАЗАТЕЛЬство. Обозначим через $\mathscr{F}_{V}$ множество гиперграней, содержающих вершину $V \in P^{n}$. Пусть $V^{*}=\prod_{F \in \mathscr{F}_{V}} v_{F}$. Мы покажем, что $V^{*}=V^{*}$ для каждых двух вершин $V, V^{\prime} \in P^{n}$. Для этого достаточно предположить, что вершины соединены ребром $V V^{\prime}$. В этом случае имеем $\mathscr{F}_{V^{\prime}}=\mathscr{F} V \backslash\{G\} \cup\left\{G^{\prime}\right\}$ для единственных гиперграней $G$ и $G^{\prime}$, окрашенных в один цвет. Если $h(G)=$ $h\left(G^{\prime}\right)=i$, умножим на $\prod_{F \in \mathscr{F}_{V} \cap \mathscr{F}_{V^{\prime}}} v_{F}$ соотношение

$$
\sum_{j=1}^{i-1} u_{j}+\sum_{F \in \mathscr{F}_{i}} v_{F}=0 .
$$

Из предложения 1 получаем

$$
\left(u_{1}+\cdots+u_{i-1}\right) \prod_{F \in \mathscr{F}_{V} \cap \mathscr{F}_{V^{\prime}}} v_{F}+V^{*}+V^{\prime *}=0 .
$$

В случае $i=1$ мы получили требуемое равенство $V^{*}=V^{\prime *}$. В случае $i>1$ для любого $1 \leqslant j<i$ имеем из предложений 1 и 2

$$
u_{j} \prod_{F \in \mathscr{F}_{V} \cap \mathscr{F}_{V^{\prime}}} v_{F}=\left\{\begin{array}{l}
0, \\
\left(u_{1}+\cdots+u_{j-1}\right) \prod_{F \in \mathscr{F}_{V} \cap \mathscr{F}_{V^{\prime}}} v_{F}, \quad \text { если } H_{j} \notin \mathscr{F}_{V} \cap \mathscr{F}_{V^{\prime}} \\
\text { если } H_{j} \in \mathscr{F}_{V} \cap \mathscr{F}_{V^{\prime}} .
\end{array}\right.
$$


Тогда по индукции следует, что $u_{j} \prod_{F \in \mathscr{F}_{V} \cap \mathscr{F}_{V^{\prime}}} v_{F}=0, j=1, \ldots, i-1$, откуда снова $V^{*}=V^{*}$.

Предположим теперь, что $u_{1} \cdots u_{n}=0$. Тогда из доказаного следует, что $V^{*}=0$ для каждой вершины $V \in P^{n}$. Более того, в этом случае все $d n$-мерные классы когомологий исчезают, потому что по следствию 2 одночлены степени $n$, свободные от квадратов, линейно порождают $H^{d n}\left(M^{d n} ; \mathbb{Z}_{2}\right)$. Но это противно известному факту, что $H^{d n}\left(M^{d n} ; \mathbb{Z}_{2}\right) \simeq \mathbb{Z}_{2}$.

2.3. Классы Штифеля-Уитни. Из предложения 1 следует, что полный класс Штифеля-Уитни можно представить в виде

$$
w\left(M^{d n}\right)=\prod_{i=1}^{n} \prod_{F \in \mathscr{F}_{i}}\left(1+v_{F}\right)=\prod_{i=1}^{n}\left(1+\sum_{F \in \mathscr{F}_{i}} v_{F}\right) .
$$

Применяя (2.1), получаем

$$
w\left(M^{d n}\right)=\left(1+u_{1}\right)\left(1+u_{1}+u_{2}\right) \cdots\left(1+u_{1}+u_{2}+\cdots+u_{n-1}\right) .
$$

С целью доказательства главной теоремы 3 воспользуемся другим множеством образующих $t_{1}, \ldots, t_{n}$, определенных следующим образом:

$$
t_{i}=\sum_{j=1}^{i} u_{j}, \quad i=1, \ldots, n .
$$

Следовательно, имеем

$$
w\left(M^{d n}\right)=\left(1+t_{1}\right) \cdots\left(1+t_{n-1}\right)
$$

По предложению 2 классы $t_{1}^{2}, t_{2}^{2}+t_{1} t_{2}, \ldots, t_{n}^{2}+t_{n-1} t_{n}$ исчезают. Обозначим через $\mathscr{T}_{n}$ порожденный ими идеал. По предложению 3 класс $t_{1} t_{2} \cdots t_{n}$ также является фундаментальным классом.

\section{§ 3. Доказательство теоремы 3}

Для доказательства теоремы 3 достаточно показать, что наивысший нормальный класс Штифеля-Уитни $\bar{w}_{d(n-1)}\left(M^{d n}\right)$ ненулевой.

3.1. Нормальные классы Штифеля-Уитни. Классы Штифеля-Уитни и нормальные классы Штифеля-Уитни связаны соотношением

$$
w\left(M^{d n}\right) \cdot \bar{w}\left(M^{d n}\right)=1
$$

Из соотношения (2.3) получаем

Лемма 2. Полный класс Штифеля-Уитни $\bar{w}\left(M^{d n}\right)$ выражается следующим образом:

$$
\bar{w}\left(M^{d n}\right)=\left(1+t_{1}\right)\left(1+t_{2}+t_{2}^{2}\right) \cdots\left(1+t_{n-1}+\cdots+t_{n-1}^{n-1}\right) .
$$


ДокАЗАТЕльство. Утверждение вытекает из того, что $t_{k}^{k+1}=0$ для всех $k=1, \ldots, n-1$. На самом деле, $t_{k}^{k+1}=\left(u_{1}+\cdots+u_{k}\right)^{k+1}$ является суммой одночленов вида $\sum u_{i_{1}}^{r_{1}}+\cdots+u_{i_{j}}^{r_{j}}$, где $1 \leqslant i_{1}<\cdots<i_{j} \leqslant k$ и $r_{1}+\cdots+r_{k}=$ $k+1$. В свою очередь, эта сумма по следствию 2 и предложению 2 является суммой однородных многочленов степени $k+1$ вида $\sum Q_{k+1}\left(u_{1}, \ldots, u_{k}\right)$. Но так как одночлены каждого многочлена в сумме свободны от квадратов, имеем $Q_{k+1}\left(u_{1}, \ldots, u_{k}\right)=0$.

Мы хотим определить наивысший ненулевой класс $\bar{w}_{k}\left(M^{d n}\right)$. Для малых $n$ вычислим $\bar{w}\left(M^{d n}\right)$ непосредственно.

ПримеР 1. 1) $\bar{w}\left(M^{d \cdot 2}\right)=1+t_{1}$;

2) $\bar{w}\left(M^{d \cdot 3}\right)=1+\left(t_{1}+t_{2}\right)$;

3) $\bar{w}\left(M^{d \cdot 4}\right)=1+\left(t_{1}+t_{2}+t_{3}\right)+t_{1} t_{3}+t_{1} t_{2} t_{3}$;

4) $\bar{w}\left(M^{d \cdot 5}\right)=1+\left(t_{1}+t_{2}+t_{3}+t_{4}\right)+\left(t_{1} t_{3}+t_{1} t_{4}+t_{2} t_{4}\right)+\left(t_{1} t_{2} t_{3}+t_{2} t_{3} t_{4}\right)$.

3.2. Класс $\bar{w}_{d(n-1)}\left(M^{d n}\right)$. Пусть $M^{d n}$ и $M^{d(n+1)}$ - отмеченные многообразия, построенные, как в п. 2.1, из простых многогранников $P_{1}^{n}$ и $P_{2}^{n+1}$, правильно окрашенных в $n$ и $n+1$ цвет соответственно.

ЛЕмма 3. Колъцо $\mathbb{Z}_{2}\left[t_{1}, \ldots, t_{n}\right] / \mathscr{T}_{n}$ является подкольцом в кольце когомологий $H^{*}\left(M^{d n} ; \mathbb{Z}_{2}\right)$, порожденным элементами $t_{1}, \ldots, t_{n}$.

ДокАЗАтельство. Необходимо доказать, что $\mathscr{T}_{n}$ - это идеал всех соотношений между элементами $t_{1}, \ldots, t_{n}$ в $H^{*}\left(M^{d n} ; \mathbb{Z}_{2}\right)$. Кольца $\mathbb{Z}_{2}\left[t_{1}, \ldots, t_{n}\right] / \mathscr{T}_{n}$ и $\mathbb{Z}_{2}\left[u_{1}, \ldots, u_{n}\right] / \mathscr{U}_{n}$ изоморфны, где $\mathscr{U}_{n}$ - идеал, порожденный элементами $u_{1}^{2}$ и $u_{i}^{2}+\left(u_{1}+\cdots+u_{i-1}\right) u_{i}, i=2, \ldots, n$, из предложения 2. Достаточно доказать, что $\mathscr{U}_{n}$ - это идеал всех соотношений между элементами $u_{1}, \ldots, u_{n}$ в $H^{*}\left(M^{d n} ; \mathbb{Z}_{2}\right)$.

По следствию 2 каждый однородный многочлен в $H^{*}\left(M^{d n} ; \mathbb{Z}_{2}\right)$ выражается как сумма мономов, свободных от квадратов. Итак, пусть у нас есть соотношение

$$
\sum_{j=1}^{m} u_{I_{j}}=0
$$

где $I_{j}=\left\{a_{e q 2.1}<\cdots<a_{j k}\right\} \subset\{1, \ldots, n\}, j=1, \ldots, m$, и $u_{I}=\prod_{i \in I} u_{i}$. Легко убедиться, что $u_{1} \cdots u_{i-1} u_{i}^{2}=0, i=1, \ldots, n$. Упорядочим множества лексикографически $I_{1}<\cdots<I_{m}$. Пусть $I_{m}=\left\{a_{1}<\cdots<a_{k}\right\}$ и $d_{i}=a_{i}-a_{i-1}$, $i=1, \ldots, k$, где $a_{0}=0$. Определим элементы

$$
U_{i}= \begin{cases}u_{a_{i-1}+1} \cdots u_{a_{i}-1}, & d_{i}>1 \\ 1, & d_{i}=1\end{cases}
$$

где $i=1, \ldots, k$. Умножая соотношение на $U_{1} \cdots U_{k}$, получаем $u_{1} \cdots u_{a_{k}}=0$, что противречит тому, что $u_{1} \cdots u_{n}$ - фундаментальный класс.

Выполняя сокращения в равенстве из леммы 2 , получаем, что каждый класс $\bar{w}_{d k}\left(M^{d n}\right)$ выражается в кольце $\mathbb{Z}_{2}\left[t_{1}, \ldots, t_{n}\right] / \mathscr{T}_{n}$ через многочлены $\bar{W}_{k}\left(t_{1}, \ldots\right.$, $t_{n}$ ) степени $k$, все одночлены которых свободны от квадратов. Заметим, что вложение

$$
i: \mathbb{Z}_{2}\left[t_{1}, \ldots, t_{n}\right] \rightarrow \mathbb{Z}_{2}\left[t_{1}, \ldots, t_{n}, t_{n+1}\right]
$$


производит естественный мономорфизм

$$
i^{*}: \mathbb{Z}_{2}\left[t_{1}, \ldots, t_{n}\right] / \mathscr{T}_{n} \rightarrow \mathbb{Z}_{2}\left[t_{1}, \ldots, t_{n}, t_{n+1}\right] / \mathscr{T}_{n+1},
$$

который в силу леммы 3 позволяет рассматривать полный класс ШтифеляУитни $\bar{w}\left(M^{d n}\right)$ как элемент кольца $H^{*}\left(M^{d(n+1)} ; \mathbb{Z}_{2}\right)$. Полные классы Штифеля-Уитни $\bar{w}\left(M^{d n}\right)$ и $\bar{w}\left(M^{d(n+1)}\right)$ удовлетворяют следующему отношению в $H^{*}\left(M^{d(n+1)} ; \mathbb{Z}_{2}\right)$ :

$$
\bar{w}\left(M^{d(n+1)}\right)=\bar{w}\left(M^{d n}\right)\left(1+t_{n}+\cdots+t_{n}^{n}\right) .
$$

В явном виде

$$
\bar{w}_{d k}\left(M^{d(n+1)}\right)=\bar{w}_{d k}\left(M^{d n}\right)+t_{n} \bar{w}_{d(k-1)}\left(M^{d n}\right)+\cdots+t_{n}^{k}, \quad k=0, \ldots, n .
$$

Напомним, что всегда $\bar{w}_{d n}\left(M^{d n}\right)=0$ (см. [10]), откуда

$$
\bar{w}_{d n}\left(M^{d(n+1)}\right)=t_{n} \bar{w}_{d(n-1)}\left(M^{d n}\right)+\cdots+t_{n}^{n}=t_{n} \bar{w}_{d(n-1)}\left(M^{d(n+1)}\right) .
$$

Мы используем тот же прием, что и в [8]. Определим числа $\sigma_{n}^{k}, 0 \leqslant k \leqslant n-1$, следующим образом

$$
\sigma_{n}^{k}=\bar{W}_{k}(\underbrace{1, \ldots, 1}_{n}) \quad(\bmod 2) .
$$

Из (3.1) и (3.2) имеем $\sigma_{n+1}^{k}=\sum_{i=0}^{k} \sigma_{n}^{i}$ для всех $k=1, \ldots, n-1$ и $\sigma_{n+1}^{n}=\sigma_{n+1}^{n-1}$. По определению чисел $\sigma_{n}^{k}$, если $\sigma_{n}^{k}=1$, то $\bar{w}_{d k}$ является суммой нечетного числа линейно независимых одночленов без квадратов, так что $\bar{w}_{d k}\left(M^{d n}\right) \neq 0$. Легко убедиться по индукции, что

$$
\sigma_{n}^{k} \equiv\left(\begin{array}{c}
n+k \\
k
\end{array}\right) \quad(\bmod 2) .
$$

В частности, если $n=2^{r}$, получаем

$$
\sigma_{n}^{n-1} \equiv\left(\begin{array}{c}
2^{r}+\left(2^{r}-1\right) \\
2^{r}-1
\end{array}\right) \equiv\left(\begin{array}{c}
2^{r+1}-1 \\
2^{r}-1
\end{array}\right) \equiv 1 \quad(\bmod 2) .
$$

Следовательно,

$$
\bar{w}_{d(n-1)}\left(M^{d n}\right)=t_{1} t_{2} \cdots t_{n-1} \neq 0
$$

откуда из теоремы 1 получаем требуемые ограничения

$$
\operatorname{imm}\left(M^{d n}\right) \geqslant d(2 n-1), \quad \operatorname{em}\left(M^{d n}\right) \geqslant d(2 n-1)+1 .
$$

Для малого накрытия $M^{n}$ из теоремы Уитни имеем $\operatorname{imm}\left(M^{n}\right)=2 n-1$ и $\operatorname{em}\left(M^{n}\right)=2 n$. Квазиторическое многообразие $M^{2 n}$ ориентируемо и поэтому вкладывается в $\mathbb{R}^{4 n-1}$. Из леммы 2 вытекает, что $\bar{w}_{2}\left(M^{2 n}\right)=t_{1}+t_{2}+\cdots+t_{n-1}$, откуда следует, что характеристический класс $\bar{w}_{2}\left(M^{d n}\right) \cdot \bar{w}_{2 n-2}\left(M^{2 n}\right)$ исчезает. Если $n \geqslant 3$, из результата Масси [11; теорема V] выводим

$$
\operatorname{imm}\left(M^{2 n}\right)=4 n-2,
$$

чем заканчиваем доказательство теоремы 3. 


\section{§4. Доказательство теоремы 4}

Пусть $n=2^{r_{1}}+2^{r_{2}}+\cdots+2^{r_{t}}, r_{1}>r_{2}>\cdots>r_{t} \geqslant 0,-$ двоичная запись числа $n$ и $m_{0}=0, m_{i}=2^{r_{i}}, i=1, \ldots, t$. Пусть дальше $P^{n}$ - простой $n$-многогранник

$$
P^{n}=P_{1}^{2^{r_{1}}} \times \cdots \times P_{t}^{2^{r_{t}}}
$$

где каждый $P_{i}^{2^{r_{i}}}$ является $2^{r_{i}}$-окрашенным простым $2^{r_{i}}$-многогранником. Очевидно, что $P^{n}$ также $n$-окрашенный.

В $\S 3$ мы построили многообразия $M^{d 2^{r_{i}}}$ над многогранниками $P_{i}^{2^{r_{i}}}$. Следуя [2; замечание, гл. 6.4], имеем, что $M^{d n}=M^{d 2^{r_{1}}} \times \cdots \times M^{d 2^{r_{t}}}$ является $G_{d^{-}}^{n^{-}}$ многообразием над $P^{n}=P_{1}^{2^{r_{1}}} \times \cdots \times P_{t}^{2^{r_{t}}}$. Полный класс Штифеля-Уитни многообразия $M^{d n}$ легко вычисляется, пользуясь следующей формулой (см. [5; задача $4 . \mathrm{A}])$ :

$$
w\left(M^{d n}\right)=w\left(M^{d 2^{r_{1}}}\right) \cdots w\left(M^{d 2^{r_{t}}}\right) \in H^{*}\left(M^{d n}\right) \cong H^{*}\left(M^{d 2^{r_{1}}}\right) \otimes \cdots \otimes H^{*}\left(M^{d 2^{r_{t}}}\right) .
$$

Соответствующий полный нормальный класс Штифеля-Уитни выражается чеpe3

$$
\bar{w}\left(M^{d n}\right)=\bar{w}\left(M^{d 2^{r_{1}}}\right) \cdots \bar{w}\left(M^{d 2^{r_{t}}}\right) .
$$

Пусть $\operatorname{rank} w(M):=\max \left\{k \mid w_{k}(M) \neq 0\right\}$. Тогда из (4.1) имеем

$$
\operatorname{rank} \bar{w}\left(M^{d n}\right)=\sum_{i=1}^{t} \operatorname{rank} \bar{w}\left(M^{d 2^{r_{i}}}\right)=\sum_{i=1}^{t} d\left(2^{r_{i}}-1\right)=n d-\alpha(n) d .
$$

Таким образом, теорема 4 вытекает из теорем 1 и 2.

Авторы выражают особую благодарность Т. Е. Панову и А. А. Гайфуллину за внимание к работе, а также рецензентам за полезные замечания.

\section{Список литературы}

[1] Г. М. Циглер, Теория многогранников, МЦНМО, М., 2014, 568 с.; пер. с англ.: G. M. Ziegler, Lectures on polytopes, Grad. Texts in Math., 152, Springer-Verlag, New York, 1995, x+370 pp.

[2] В. М. Бухштабер, Т. Е. Панов, Торические действия в топологии и комбинаторике, МЦНМО, М., 2004, 272 с.

[3] M. Joswig, "Projectivities in simplicial complexes and colorings of simple polytopes", Math. Z., 240:2 (2002), 243-259.

[4] M. W. Davis, T. Januszkiewicz, "Convex polytopes, Coxeter orbifolds and torus actions", Duke Math. J., 62:2 (1991), 417-451.

[5] Дж. Милнор, Дж. Сташеф, Характеристические классы, Мир, М., 1979, 371 с.; пер. с англ.: J.W. Milnor, J.D. Stasheff, Characteristic classes, Ann. of Math. Stud., 76, Princeton Univ. Press, Princeton, NJ; Univ. of Tokyo Press, Tokyo, 1974, vii+331 pp.

[6] M. Ghomi, S. Tabachnikov, "Totally skew embeddings of manifolds", Math. Z., 258:3 (2008), 499-512.

[7] D. Baralić, B. Prvulović, G. Stojanović, S. Vrećica, R. Živaljević, "Topological obstructions to totally skew embeddings", Trans. Amer. Math. Soc., 364:4 (2012), $2213-2226$. 
[8] D. Baralić, "Immersions and embeddings of quasitoric manifolds over the cube", Publ. Inst. Math. (Beograd) (N.S.), 95:109 (2014), 63-71.

[9] R. L. Cohen, "The immersion conjecture for differentiable manifolds", Ann. of Math. (2), 122:2 (1985), 237-328.

[10] W. S. Massey, "On the Stiefel-Whitney classes of a manifold", Amer. J. Math., 82:1 (1960), 92-102.

[11] W.S. Massey, "Normal vector fields on manifolds", Proc. Amer. Math. Soc., 12 (1961), 33-40.

Джордже Б. Баралич

(Djordje B. Baralić)

Mathematical Institute,

Serbian Academy of Sciences and Arts,

Belgrade, Serbia

E-mail: djbaralic@mi.sanu.ac.rs

\section{Владимир Н. Груйич}

(Vladimir N. Grujić)

Faculty of Mathematics,

University of Belgrade, Serbia

E-mail: vgrujic@matf.bg.ac.rs
Поступила в редакцию 08.07.2014 и 26.12.2015 